\title{
Visible Light-Assisted Photoreduction of Graphene Oxide Using CdS Nanoparticles and Gas Sensing Properties
}

\author{
Amirhossein Hasani, ${ }^{1}$ Hamed Sharifi Dehsari, ${ }^{2}$ Ali Amiri Zarandi, ${ }^{2}$ Alireza Salehi, ${ }^{1}$ \\ Faramarz Afshar Taromi, ${ }^{2}$ and Hanif Kazeroni ${ }^{3}$ \\ ${ }^{1}$ Department of Electrical Engineering, K.N. Toosi University of Technology, Seyyed Khandan, Tehran 16317-14191, Iran \\ ${ }^{2}$ Department of Polymer Engineering and Color Technology, Amirkabir University of Technology, No. 424 Hafez Street, \\ Tehran 15875-4413, Iran \\ ${ }^{3}$ Department of Chemical Engineering, Amirkabir University of Technology, No. 424 Hafez Street, Tehran 15875-4413, Iran
}

Correspondence should be addressed to Alireza Salehi; salehi@kntu.ac.ir and Faramarz Afshar Taromi; afshar@aut.ac.ir

Received 12 September 2014; Revised 20 December 2014; Accepted 22 December 2014

Academic Editor: Joydeep Dutta

Copyright (C) 2015 Amirhossein Hasani et al. This is an open access article distributed under the Creative Commons Attribution License, which permits unrestricted use, distribution, and reproduction in any medium, provided the original work is properly cited.

Graphene oxide sheets suspended in ethanol interact with excited CdS nanoparticles and contributed to photocatalytic reduction by accepting electron from nanoparticle. The UV-Vis measurement showed that electrical absorbance of the CdS/graphene oxide sheets increased by decreasing the irradiation time and after $2 \mathrm{~h}$ it remained constant which indicates the optimum reduction time. Furthermore, the direct interaction between CdS nanoparticles and graphene sheets hinders the collapse of exfoliated sheets of graphene. The 4-point probe measurement of nanocomposite with different ratios of graphene oxide in CdS solution after irradiation shows that the conductivity of them increased by increasing the amount of GO, but further increasing causes incomplete photo reduction process due to exorbitance increasing GO sheets which contribute to decreasing the conductivity. The CdS/RGO composite material can be used as a gas sensor for $\mathrm{CO}_{2}$ based on its electrocatalytic behavior. The low-cost and easy fabrication sensor shows rapid response and high sensitivity. By varying the amount of GO the optimum concentration which shows high sensitivity is found and its good performance compared with other is attributed to its higher conductivity due to complete reduction. Moreover, the effects of thermal annealing on the conductivity of CdS/RGO film and the performance of devices are researched.

\section{Introduction}

Graphene is two-dimensional nanomaterial consisting of a single layer of $\mathrm{sp}^{2}$ network of carbon atoms [1]. While the thickness of a graphene sheet is on the order of a single atomic unit, its lateral dimension can reach up to tens of microns. Graphene derivatives like graphene oxide (GO) and reduced graphene oxide (RGO, chemically modified graphene) have attracted great interests in electronics, optoelectronics, display devices, and sensing devices due to their remarkably high electron mobility, extremely large surface area, high mechanical ability, and low fabrication cost [2-4]. Sensing devices have been widely studied with great results because graphene's 2D crystal structure allows every carbon atom a surface atom so that electron transport through graphene can be highly sensitive to adsorbed molecules which can be demonstrated down to a single molecular level [5]. Several methods have been reported to produce graphene, such as mechanical exfoliation [1], graphitization of $\mathrm{SiC}$ [6], CVD (Chemical Vapor Deposition) growth on metal substrate [7], and reduction of graphene oxide [8]. Most of graphene used in electronic devices is produced with the last method of GO reduction due to its easy synthesis method and low cost material. The graphene in a graphene-based composite is often prepared by the reduction of graphene oxide which is commonly referred to as reduced graphene oxide (RGO). Meanwhile, reduction of graphene oxide (GO) has been widely used to produce graphene, such as the chemical reduction of GO [9], thermal reduction of GO [10], flash reduction of $\mathrm{GO}$ under $\mathrm{N}_{2}$ atmosphere [11], direct laser writing on GO sheets [12], and conductive atomic force microscope tip-based electrochemical reduction of GO [13]. 
However, all these methods have intrinsic outcome, like the existence of toxic chemicals like hydrazine, requiring rapid heating processes and high reaction temperatures, special instruments, or expensive high-energy light sources, and so forth, which limit their practical applications.

Photocatalytic reduction of GO has been recently proven to be an effective method to produce graphene by Williams and coworkers [14]. Compared to conventional chemical reduction, the photoreduction of GO is "ecofriendly" and it can be controlled easily via the irradiation properties, but it requires semiconductor nanoparticles with large band gaps such as $\mathrm{TiO}_{2}$ and $\mathrm{ZnO}$, used as photocatalysts, to absorb light [15]. Also it recently reported a novel method for photoreduction of graphene oxide by $\mathrm{Ag}$ nanoparticles as a plasmonic photocatalyst [15]. Semiconductor nanoparticles, especially cadmium sulfide (CdS) nanoparticles, have been studied in the last few years, due to their potential applications based on size-tunable optical properties and high mobility [1619]. CdS has been generally considered as a strong candidate for high efficiency visible-light-driven photocatalysts. A combination of graphene, exhibiting exceptionally high carrier mobility, and CdS nanostructures with size-tunable optical properties offers interesting optoelectronic applications [20-22]. Arising from the inhibited recombination rate of electron-hole pairs in graphene-based composites, much attention has been paid to the photocatalytic application of CdS/RGO composites such as solar cells [20, 23, 24], fuel cells $[25,26]$, light-emitting diodes [20], and lasers and sensors $[22,23]$ which contribute to the improvement in their performance by increasing carrier mobility.

Undoubtedly, the increased active surface areas and high carrier mobility also make graphene-based nanocomposites perfect materials for high-performance sensing devices $[27,28]$. In particular, the search for alternative low-cost graphene-based biosensors to traditional noble metal-based sensors is the beginning of this excellent topic [29]. For example, CdS nanorods were immobilized on graphene to construct a mediator-free $\mathrm{H}_{2} \mathrm{O}_{2}$ sensor, with a detection range of $0.1 \mathrm{mM}$ to $0.5 \mathrm{mM}$. Even so, novel graphene-based gas sensors are extremely desirable as they are low cost and show rapid and highly sensitive response. To the best of our knowledge, little attention has been paid to the electrochemical application of CdS/RGO composites as high performance gas sensors [30].

Carbon dioxide $\left(\mathrm{CO}_{2}\right)$ is one of the most important greenhouse gases which causes a phenomenon known as global warming. Efforts are being done in order to monitor and subsequently reduce $\mathrm{CO}_{2}$ emission from the combustion of fuel in vehicles and the burning of coal in industries. Therefore, it is crucial to make low-cost, sensitive, adjustable sensors that can be used to monitor the $\mathrm{CO}_{2}$ concentration in industrial exhaust gases [31]. Several research groups have been attempting to make improvements in gas sensors, with the aim of enhancing the sensing performance. For the past several years, graphene-based composites have been considered promising candidates for sensing materials that can detect extremely low concentrations (ppb range) of gases such as $\mathrm{NO}_{2}, \mathrm{NH}_{3}, \mathrm{O}_{2}, \mathrm{H}_{2}, \mathrm{CO}_{2}$, and $\mathrm{CO}$ [31-38]. The high sensitivity of graphene-based gas sensors can be attributed to the fantastic electrical properties, small size, high surface area, and large gas-adsorption capacity of graphene-based composite.

In previous work, the synthesis of ultra large graphene oxide sheets based on a novel solution-phase method involving preexfoliation of graphite was reported [39]. In this work, CdS nanoparticle and GO were synthesized separately and $\mathrm{CdS} / \mathrm{RGO}$ composites are fabricated through mixing the solutions and the photoreduction of composite under visible irradiation for 2 hours. Then considering the large specific area and high carrier mobility, CdS nanoparticle/RGO composites are used to fabricate a sensing device for $\mathrm{CO}_{2}$. For fabrication of the sensing devices, CdS/GO solution after irradiation with various amounts (CdS/GO (1\%), CdS (3\%), and CdS/GO (5\%)) is drop-casted onto Al electrode with silicon substrate for comparison and finding the optimum one. Furthermore, we investigate the effect of thermal annealing as a posttreatment of CdS/RGO films. Thermal annealing is performed using an annealing oven with $\mathrm{N}_{2}$ atmosphere at $300^{\circ} \mathrm{C}$. FTIR, Uv-Vis, XRD, and 4-point probe are used to characterize the reduction of graphene oxide. The gas sensors based on present $\mathrm{CdS} / \mathrm{RGO}$ composite showed capability of detecting $\mathrm{CO}_{2}$ at a level of parts-per-million (ppm) concentration with high sensitivity and fast response.

\section{Experimental Details}

2.1. Materials. Natural graphite flakes ( 325 mesh, 99.8\%) were purchased from Alfa Aesar (America). Sulfuric acid $\left(\mathrm{H}_{2} \mathrm{SO}_{4}, 98 \%\right)$, phosphoric acid $\left(\mathrm{H}_{3} \mathrm{PO}_{4}, 85 \%\right)$, potassium permanganate $\left(\mathrm{KMnO}_{4}, 99.5 \%\right)$, peroxide $\left(\mathrm{H}_{2} \mathrm{O}_{2}, 30 \%\right)$, hydrochloric acid ( $\mathrm{HCl}, 36-38 \%)$, ethanol (99.7\%), EDA (99\%), and Rhm B (AR) were purchased from Sinopharm Chemical Reagent Co., Ltd. (Shanghai, China). Cadmium nitrate $\left(\mathrm{Cd}\left(\mathrm{NO}_{3}\right)_{2} \cdot 4 \mathrm{H}_{2} \mathrm{O}, 99 \%\right)$ was obtained from Jin Shan Ting Xin Chemical Reagent.

2.2. Synthesis of Graphene Oxide. Graphene oxide was prepared by a modified Hummers method as reported previously [40]. Briefly, concentrated $\mathrm{H}_{2} \mathrm{SO}_{4}(69 \mathrm{~mL})$ was added to a mixture of expanded graphite $(3 \mathrm{~g})$ and $\mathrm{NaNO}_{3}(1.5 \mathrm{~g})$, and the mixture was cooled to $0^{\circ} \mathrm{C}$ in an ice bath. Then $9 \mathrm{~g} \mathrm{KMnO}_{4}$ was slowly added to the mixture with stirring and cooling; therefore, the temperature of the solution was kept below $20^{\circ} \mathrm{C}$, and the temperature was then increased up to $35^{\circ} \mathrm{C}$ while stirring for $30 \mathrm{~min}$. Then, $138 \mathrm{~mL}$ of water was added slowly, because exothermic process temperature reached $98^{\circ} \mathrm{C}$. External heating was also introduced to maintain the reaction temperature at $98^{\circ} \mathrm{C}$ for $15 \mathrm{~min}$; after that, the heat was removed and the reaction was cooled using a water bath for $10 \mathrm{~min}$. After that, an additional $420 \mathrm{~mL}$ of distilled water was added to the reaction vessel, followed by the addition of $3 \mathrm{~mL}$ of $30 \% \mathrm{H}_{2} \mathrm{O}_{2}$. The solid product was separated by centrifugation in high speed and washed repeatedly with 5\% $\mathrm{HCl}$ solution and distilled water. The synthesized graphite oxide was dried in an air oven at $70^{\circ} \mathrm{C}$ overnight. To prepare graphene oxide, the synthesized graphite oxide was dispersed in distilled water to make a yellow-brown dispersion, and 

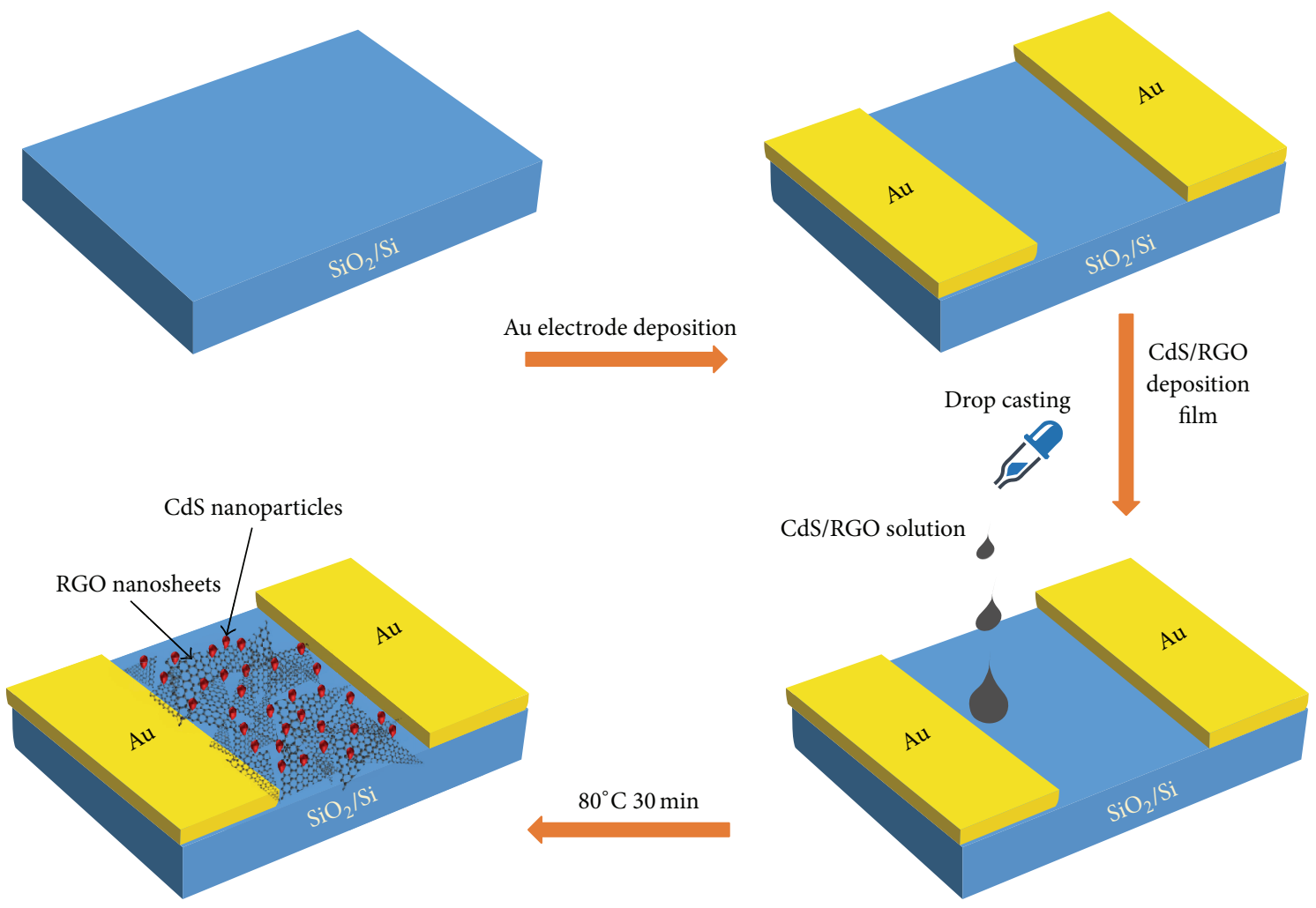

FIGURE 1: Schematic steps of gas sensor fabrication.

the exfoliation of graphite oxide to make graphene oxide was achieved by mild sonication in a bath sonicator for $30 \mathrm{~min}$.

\subsection{Synthesis of CdS Nanoparticles. Nanoparticles of CdS} were synthesized in an aqueous medium through chemical precipitation technique starting from cadmium sulfate and thiourea, using triethanolamine as complexing agent. In a typical procedure, aqueous solution of cadmium sulfate $(0.1 \mathrm{M})$ was prepared. While stirring this solution, an appropriate quantity of triethanolamine was added to obtain a stable Cd-TEA complex. The $\mathrm{pH}$ of the reaction mixture was adjusted to about 10.5 using sodium hydroxide. Then aqueous solution of thiourea was added to reaction mixture and the reaction mixture was kept in an oil bath at a suitable temperature. After $1 \mathrm{~h}$, the precipitates were harvested by centrifugation, washed several times using deionized water, and then dried in oven at $70^{\circ} \mathrm{C}$ for $12 \mathrm{~h}$.

\subsection{Visible Light-Assisted Reduction of Graphene Oxide in CdS} Suspension. In this work, we prepared a mixture of graphene oxide and colloidal CdS nanoparticles. $14 \mathrm{mg}$ of graphene oxide was dispersed in $7 \mathrm{~mL}$ ethanol under ultrasonic dissolved (TECNO-GAZ, S.P.A, Italy) method for $40 \mathrm{~min}$ to form a graphene oxide solution. A homogeneous brown GO solution was obtained, and no particle was observed. CdS colloidal suspension $(10 \mathrm{mM})$ in ethanol was prepared for comparison, and different amount of GO (CdS/GO- $1 \mathrm{wt} \%$, CdS/GO-3 wt\%, CdS/GO-5 wt\%, and CdS/GO-50 wt\%) was added to the solution, stirred, and sonicated for $60 \mathrm{~min}$ to produce dispersion of graphene oxide sheets coated with the $\mathrm{CdS}$ nanoparticles. Visible irradiation of the CdS-graphene oxide (CdS-GO) samples was performed using home-made reactor equipped with a $300 \mathrm{~W}$ halogen lamp. The samples were subjected to visible irradiation for 2 hours.

2.5. Sensor Fabrication. Investigating the $\mathrm{CO}_{2}$ gas sensing properties of visible light-assisted photocatalytic reduction of graphene oxide using CdS nanoparticles based sensor (in-plane resistive) was fabricated via a two-step procedure. The first step was to fabricate microscale Au electrodes and deposition onto silicon substrate. By the PVD method Au electrodes deposited onto silicon substrate. The thickness of $\mathrm{Au}$ electrodes is about $200 \mathrm{~nm}$ and with $50 \mu \mathrm{m}$ space of each. The next step was the fabrication of the sensor using $\mathrm{CdS} / \mathrm{GO}$ nanocomposite as the $\mathrm{CO}_{2}$-sensing material, in which drop-casting was adopted with the different solutions (CdS/GO-1\%, CdS/GO-3\%, CdS/GO-5\%, and CdS/GO-50\%) after irradiation activity. Figure 1 illustrates configuration of the sensor with the CdS/GO composite. A drop of CdS/GO solution was dripped on the Au electrodes through a pipette. Then the sensor was backed for $30 \mathrm{~min}$ in a furnace at $80^{\circ} \mathrm{C}$ in the nitrogen atmosphere. Moreover, in order to perform thermal annealing process the films are exposed to $300^{\circ} \mathrm{C}$ at $\mathrm{N}_{2}$ atmosphere.

2.6. Characterization of Materials. The crystallographic structural analysis was carried out by X-ray diffraction (XRD) method. Powder XRD patterns were taken on a Philips 


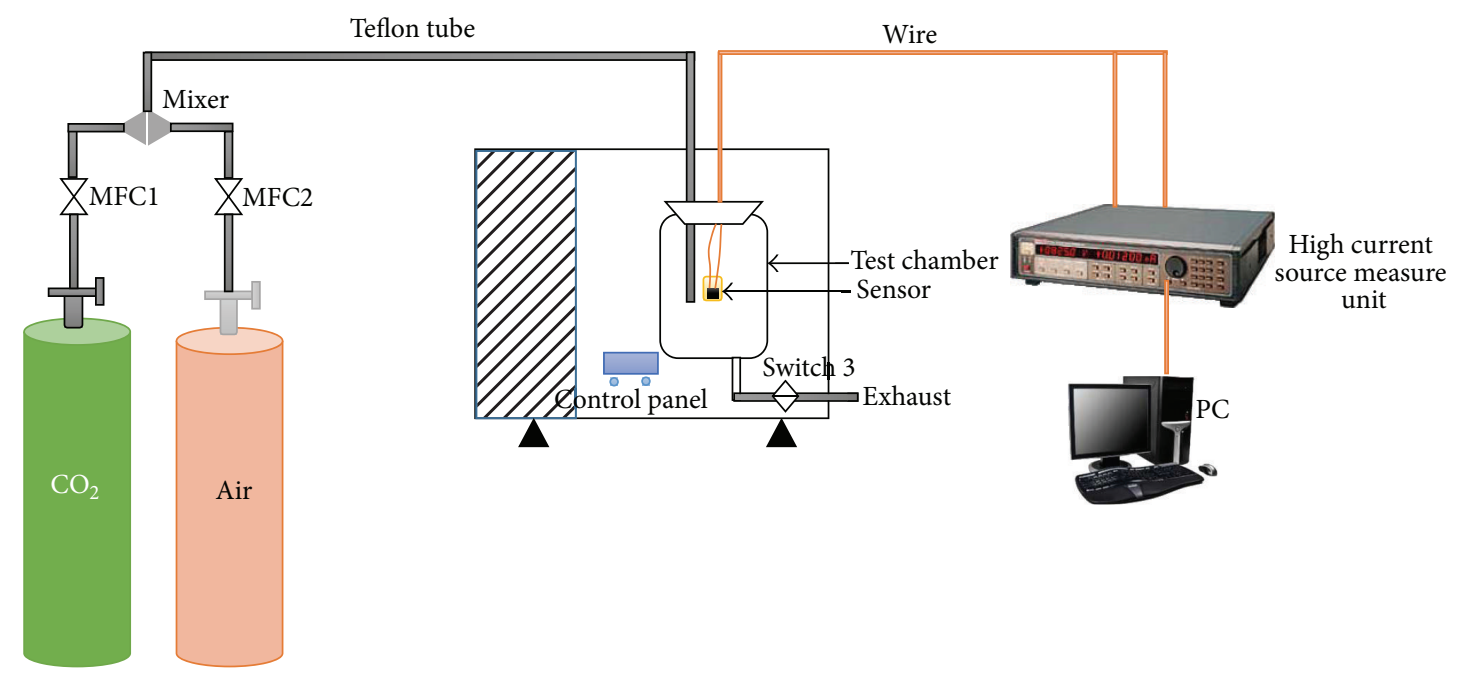

FIgURE 2: Experimental setup for measurements performed using the CdS/RGO composite-based $\mathrm{CO}_{2}$ gas sensor.

$X$ 'Pert Pro diffractometer using a Ni-filtered $\mathrm{CuK} \alpha$ radiation $(\lambda=0.154056 \mathrm{~nm})$ over the $2 \theta$ range of $0^{\circ}$ to $80^{\circ}$ and using $35 \mathrm{~mA}$ and $40 \mathrm{kV}$ current. Fourier-transformed infrared (FT-IR) spectroscopy (KBr) was collected using a Bruker, IFS-66/S over the wavenumber range of $4000-400 \mathrm{~cm}^{-1}$. For characterization of the GO, Raman spectra of the GO were observed with a Nanofinder 30 (Tokyo Instruments Co., Osaka, Japan). Scanning electron microscopy (SEM) images were taken on leo $1455 \mathrm{vp}$. Absorption spectra were taken on a UV-Vis-spectrophotometer (Perkin-Elmer, Lambda EZ201, USA). The conductivity of sensing layer was measured for electrical characterization of the composite films using a four-point probe. DekTak 8000 profilometer was used to determine the thickness of nanocomposites. The measurement of ionization potential and the electron affinity of the CdS and GO was executed using a cyclic voltammetry (CV, CH Instruments-611C) [39, 41].

2.7. Sensing Response Characterization. The $\mathrm{CO}_{2}$ gas response characteristics of the optimized CdS/RGO composites were investigated by recording their electrical responses upon exposure to flows of dry air and $\mathrm{CO}_{2}$ gas at room temperature. Gas flows containing controlled analyte vapors were injected into the chamber by a mass flow meter (Alicat scientific, Tucson, USA). Dry air was used to flush the device between exposures to $\mathrm{CO}_{2}$-containing gas flows (Figure 2). I-V measurements of sensor devices were recorded with a Keithley (238 Hight Current Source Measure Unit) multimeter and sensitivity was defined by the following equation:

$$
\text { Sensitivity }=\text { normalized current }=\frac{I_{\text {air }}}{I_{\text {gas }}} \text {, }
$$

where $I_{\text {gas }}$ is the current of the sensor when exposed to $\mathrm{CO}_{2}$ gas and $I_{\text {air }}$ is the initial current of the sensing materials when exposed to air dry at 0.2 applied voltage.

\section{Results and Discussion}

Raman spectra are commonly used to characterize graphenebased materials. The Raman spectra of the graphitic systems have two main peaks: the peak at $\sim 1575 \mathrm{~cm}^{-1}$ is the Gband, which corresponds to the planar configuration of $\mathrm{sp}^{2}$ bonded carbon that constitutes graphene, and the peak at $\sim 1352 \mathrm{~cm}^{-1}$ is the D-band, representing structural disorders defect that breaks the translational symmetry (like impurity, functionalization, structural defect, etc.) [41, 42]. Figure 3 presents the micro-Raman spectra of GO which shows the G-band and D-band of GO at approximately $1575 \mathrm{~cm}^{-1}$ and $1352 \mathrm{~cm}^{-1}$. The appearance of $\mathrm{G}$ peak of $\mathrm{GO}$ at a higher region was ascribed to the extensive oxidation of graphite and destruction of its periodic structure.

Figure 4(a) shows the SEM image of the primal sample of CdS nanoparticle with flake layer structure. SEM of the $\mathrm{CdS}$ clearly shows the formation of fine spherical particles of $\mathrm{CdS}$ which the majority size of the CdS nanoparticles was in the range of about $20 \mathrm{~nm}$. The corresponding SEM image of GO is presented in Figure 4(b). The GO sheets had a lateral size predominantly on the order of $30-70 \mu \mathrm{m}$, with many of them being even larger than $100 \mu \mathrm{m}$ and up to $300 \mu \mathrm{m}$. However, fragments of smaller GO sheets, typically $10-20 \mu \mathrm{m}$ in diameter, were also observed, which are thought to be inevitable due to breakages during the exfoliation process.

Figure 5 shows the FT-IR spectra of GO (black one), the powder of $\mathrm{CdS}$ (red one), the mixture of GO/CdS (green one), and a RGO/CdS mixture reduced by visible light (blue one) as produced using the reported method. For $\mathrm{GO}$, the characteristic peaks appear at $3422 \mathrm{~cm}^{-1}(\mathrm{O}-$ $\mathrm{H}$ stretching vibrations), $1743 \mathrm{~cm}^{-1}$ (stretching vibrations from $\mathrm{C}=\mathrm{O}$ ), $1632 \mathrm{~cm}^{-1}$ (skeletal vibrations from unoxidized graphitic domains), $1416 \mathrm{~cm}^{-1}$ (C-O carboxy), $1220 \mathrm{~cm}^{-1}$ (C$\mathrm{O}$ epoxy), and $1150 \mathrm{~cm}^{-1}$ (C-O stretching vibrations). In the $\mathrm{CdS}$ spectrum, the peak at $3420 \mathrm{~cm}^{-1}$ is assigned to $\mathrm{O}-\mathrm{H}$ stretching of absorbed water on the surface of CdS. The presence of water is confirmed by its bending vibration at 


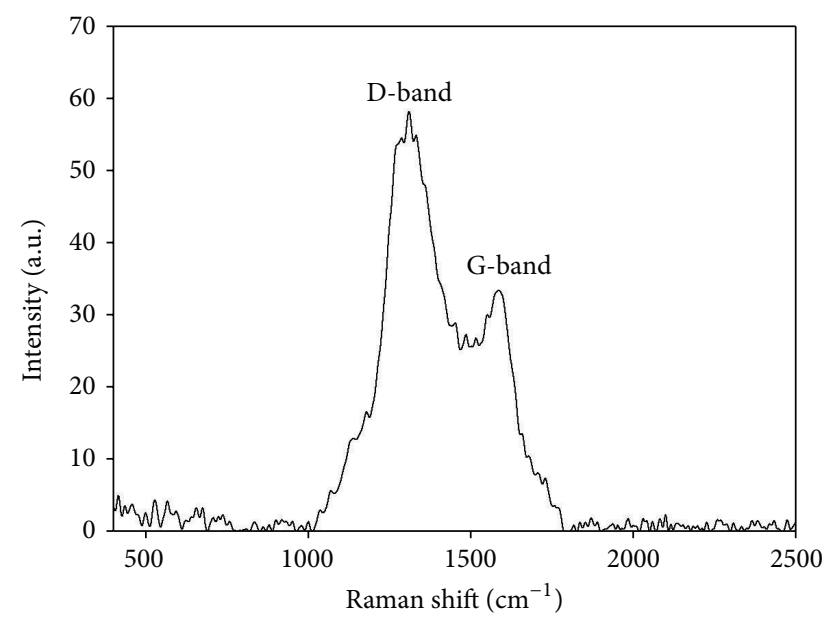

FIGURE 3: The Raman spectra of graphene oxide powder.

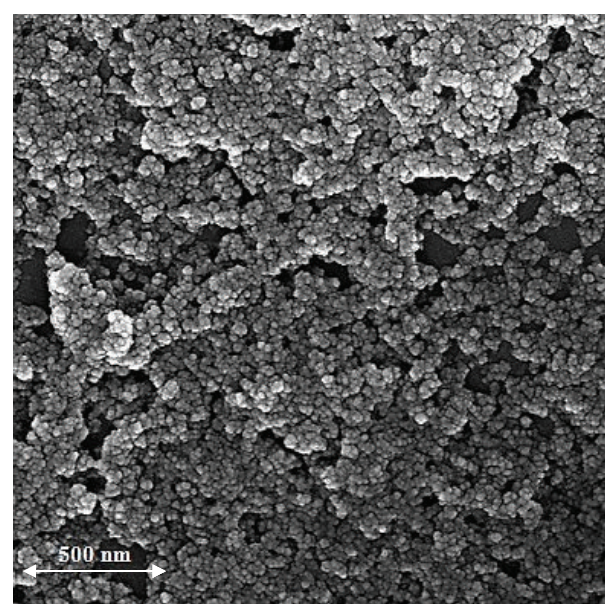

(a)

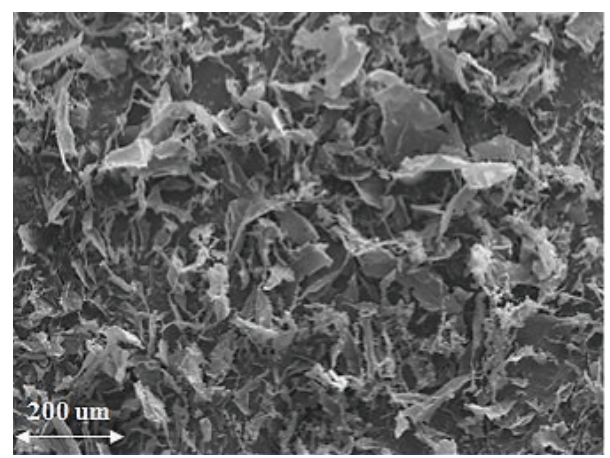

(b)

FIGURE 4: SEM micrograph of as-prepared CdS nanoparticles (a) and GO (b).

$1625 \mathrm{~cm}^{-1}$. The peak at $1402 \mathrm{~cm}^{-1}$ and $665 \mathrm{~cm}^{-1}$ due to C$\mathrm{S}, \mathrm{Cd}-\mathrm{S}$, and S-S was also detected from the FT-IR spectra. Furthermore, as can be seen from green one the presence of different types of oxygen functionalities in GO/CdS was confirmed (such as $\mathrm{O}-\mathrm{H}, \mathrm{C}=\mathrm{O}$, and $\mathrm{C}-\mathrm{O}$ ). However, in the FT-IR spectrum of $\mathrm{CdS} / \mathrm{RGO}$, the characteristic absorption

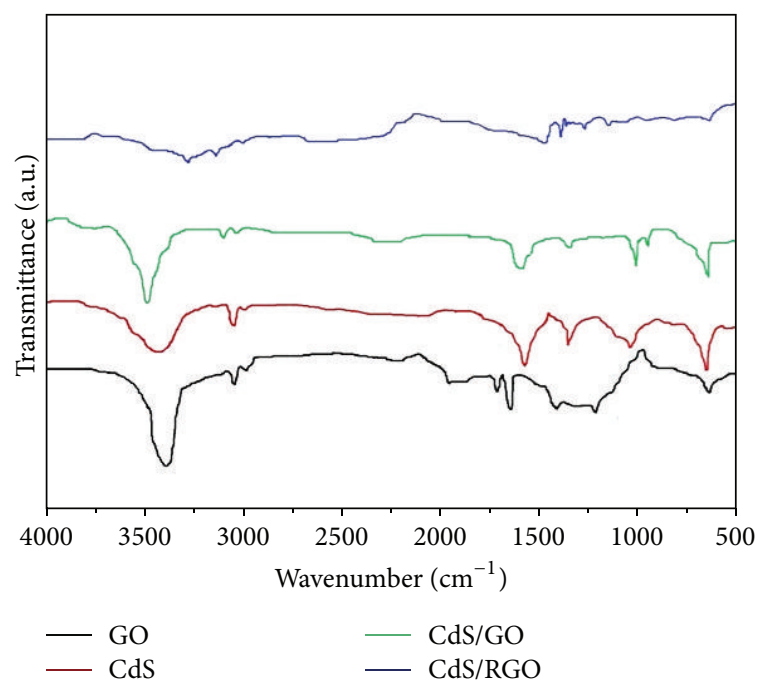

Figure 5: The FTIR spectra of GO, CdS, CdS/GO, and CdS/RGO.

bands of $\mathrm{C}=\mathrm{O}, \mathrm{C}-\mathrm{O}$, and $\mathrm{O}-\mathrm{H}$ decreased dramatically, demonstrating that $\mathrm{GO}$ has been reduced.

Ethanol suspensions of GO and $\mathrm{CdS}$ nanoparticles were exposed to visible light. The solutions were exposed to nitrogen for approximately $15 \mathrm{~min}$. The air should be removed from system since dissolved oxygen competes for the photoinduced electron; thus, it can cause the reduction of GO to be ineffective. As the CdS-GO suspension in ethanol was irradiated with visible light, the change in color from yellow to dark black was shown (Figure 6). This change in color is very similar to the color change observed during the chemical reduction of graphene oxide and it can be attributed to the reduction of graphene oxide. As discussed earlier, this color change is indicative of "partial restoration of the (conjugated) $\pi$ network" in the graphene sheets [43]. Also no such color changes could be seen if we irradiate a graphene suspension in ethanol without $\mathrm{CdS}$ which shows the vital effect of $\mathrm{CdS}$ in reducing the solution.

Figure 6(a) shows the absorption changes seen during the visible irradiation of a CdS-GO nanoparticle suspension, respectively. The inset in Figure 6(b) shows the growth of absorption at $300 \mathrm{~nm}$. The shift in absorption occurs as a consequence of the darkening of solution and is maximized after approximately $120 \mathrm{~min}$ of irradiation which shows the finishing of reduction. It should be noted that a maximized color change does not necessarily correspond to a complete reduction. Additional exposure of UV light is crucial to maximize the reduction of GO.

The photocatalytic properties of semiconducting CdS particles are well known [30]. A similar reaction was previously reported for $\mathrm{TiO}_{2}$-graphene and $\mathrm{ZnO}$-graphene composites $[43,44]$. The schematic of reaction is shown briefly in Figure 7. GO sheet is covered with mostly epoxide $(1,2-$ ether) and $\mathrm{OH}$ groups on its sheets and thus can be treated as a semiconductor with a large band gap. When CdS is exposed to visible light, it absorbed visible light considerably. Then, the photoinduced electron-hole pairs are generated 


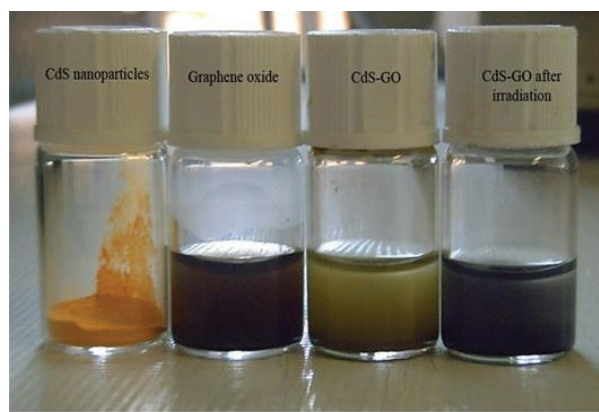

(a)

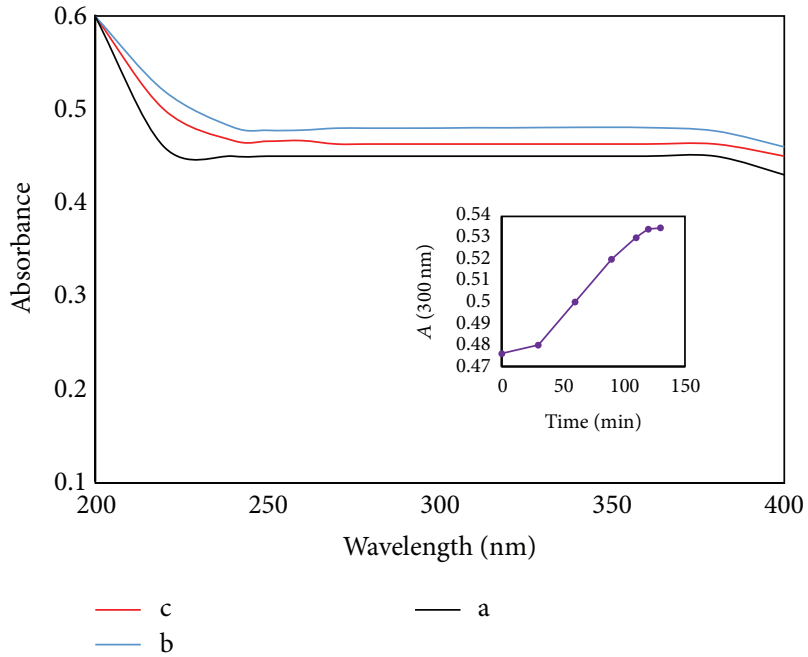

(b)

FIgURE 6: (a) The change in color of a solution of CdS nanoparticles with GO solution before and after visible-light irradiation, (b) the absorption spectra of graphene oxide and CdS suspension in ethanol, a: before visible-light irradiation, b: after 80 min, and c: after 120 min of visible-light irradiation.

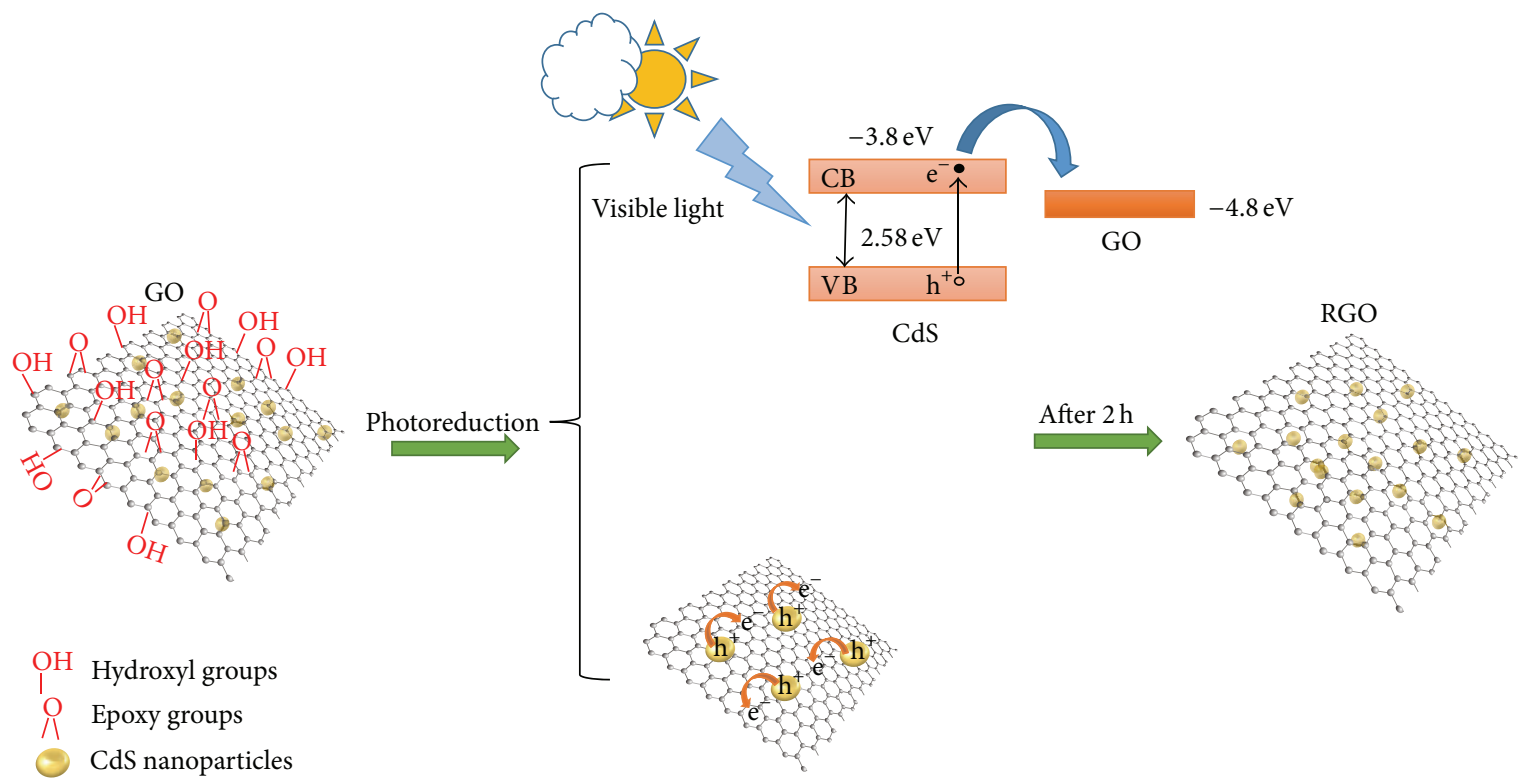

FIGURE 7: Schematic diagram of the photoreduction processes under visible-light irradiation.

which cause a great enhancement of the local electromagnetic fields near the rough CdS surface by photoexcited metallic "electrons" and "holes." In the presence of ethanol the holes are scavenged to produce ethoxy radicals, thus leaving the electrons to accumulate within CdS particles (reaction (2)). Consequently, these photoexcited electrons are injected into the conduction band of GO, leading to the reduction of the functional groups in GO as well as the oxidation of CdS NP (reaction (3)). In fact, it was previously shown that the electrons stored in CdS nanoparticles are readily scavenged by carbon nanostructures such as fullerenes and carbon nanotubes $[14,45,46]$. The work functions GO is $-4.8 \mathrm{eV}$. This value and the conductive and valance band of CdS are listed in the energy diagram shown in Figure 7. The energy barrier between GO and CdS was about $1 \mathrm{eV}$. The work function of $\mathrm{GO}$ is lower than the conductive band of CdS. Therefore, GO acts as an electron acceptor in this system:

$$
\begin{aligned}
\mathrm{CdS}+h v & \longrightarrow \mathrm{CdS}(h+\mathrm{e}) \\
& \stackrel{\text { Ethanol }}{\longrightarrow} \mathrm{CdS}(\mathrm{e})+\mathrm{C}_{2} \mathrm{H}_{4} \mathrm{OH}^{\bullet}+\mathrm{H}^{+}
\end{aligned}
$$

CdS $(\mathrm{e})+$ graphene oxide $\longrightarrow \mathrm{CdS}+$ graphene reduced 


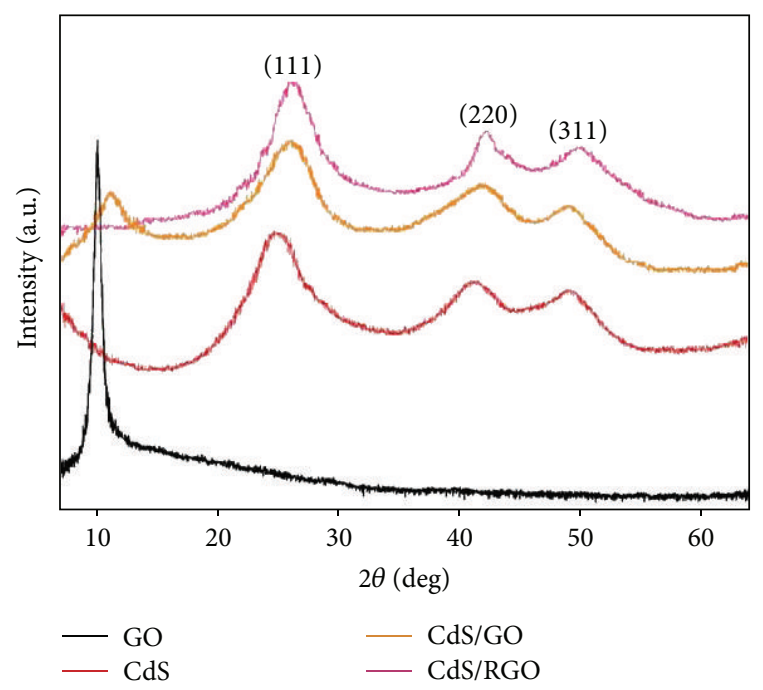

FIGURE 8: The XRD pattern of GO, CdS, CdS/GO, and CdS/RGO.

Furthermore, the ethoxy radicals produced during the hole oxidation step are also reductive in nature and thus result in the reduction process [14]. No such reductive radicals are produced in the absence of CdS. In the present experiments graphene oxide, with its oxygen groups (epoxy and carboxylate), readily interacts with CdS particles and undergoes reduction under visible irradiation. Interactions between the groups in $\mathrm{CdS}$ and the functional groups of GO (hydroxyl, carboxyl, and epoxy groups) facilitated the basis to obtain CdS-graphene composites. In addition, it enables us to explore a photocatalytic reduction procedure to obtain reduced graphene sheets in solution.

The crystallographic structural analysis was carried out by X-ray diffraction (XRD) method. Powder XRD patterns were taken on a Philips X'Pert Pro diffractometer using a Nifiltered $\mathrm{CuK} \alpha$ radiation $(\lambda=0.154056 \mathrm{~nm})$ over the $2 \theta$ range of $0^{\circ}$ to $80^{\circ}$ and using $35 \mathrm{~mA}$ and $40 \mathrm{kV}$ current. The XRD pattern of the precipitated GO was illustrated in Figure 8 (black curve). The XRD pattern of GO shows an intense and sharp peak centered at $2 \theta=10.2^{\circ}$, corresponding to a $d$ spacing of about $8.78 \AA$ according to Bragg's law: $2 d \sin \theta=$ $n \lambda$, where $n$ is an integer determined by the given order and $\lambda$ is the wavelength. The increase in interplanar distance of GO compared with the graphite is due to the existence of oxygen functional groups and some other structural defects. The XRD pattern of the precipitated nanoparticles was illustrated in Figure 8 (red curve). The CdS sample shows four broad diffraction peaks attributed to the hexagonal phase of the CdS. These broad peaks even overlap with their neighbors, indicating that the sizes of the particles are in nanorange and are poorly crystallized. Moreover, Figure 8 (orange curve) shows the XRD patterns of CdS/GO $1 \mathrm{wt} \%$ before and after reduction using visible-light irradiation. In GO the large interlayer distance has been attributed to the formation of hydroxyl, epoxy, and carboxyl groups. With reduction, the interlayer distance is expected to contract due to the removal of such functional groups. Using visible-assisted light irradiation after 2 hours, the peak of the large interlayer

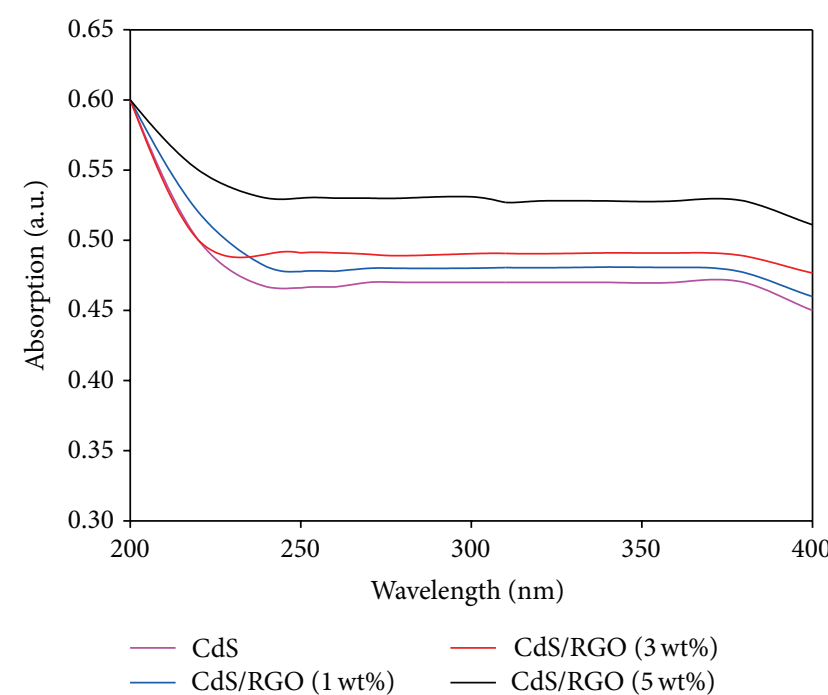

FIGURE 9: Absorption spectra of CdS/RGO composites with various percentages of RGO (1\%, 3\%, and 5\%) and CdS.

distance is removed from $2 \theta=10.2$; see Figure 8 (pink curve). This shows that most of the functional groups in GO were removed and it confirms that the graphene oxide was reduced using CdS nanoparticle and visible-light irradiation.

In order to further study, UV-vis absorption spectra were employed to investigate the optical properties of the CdS/RGO composites. As shown in Figure 9, CdS nanoparticles display a shoulder around $480 \mathrm{~nm}$, indicating that the band edge is about $2.58 \mathrm{eV}$, which is close to the band gap energy of CdS crystal $(2.42 \mathrm{eV})$. Compared with pure CdS, as we expected, the CdS/RGO exhibits a CdS absorption peak and enhanced absorption intensity especially in the visiblelight region with the increase of GO; their scattering also was enhanced, which is due to the slope changes in the region ranging from $600 \mathrm{~nm}$ to $800 \mathrm{~nm}$.

In Figure 10 the 4-point probe conductivity measurements of as-prepared films with different ratio of graphene oxide in CdS solution, before and after UV-Vis photoreduction and annealing, are shown. The thickness of films was approximately $\sim 10 \mu \mathrm{m}$. As it can be seen from Figure 10, the conductivity of CdS/GO composite ( $5 \mathrm{wt} \%$ ) film before visible-light irradiation is very low, though the conductivity of films with $1 \mathrm{wt} \%, 3 \mathrm{wt} \%$, and $5 \mathrm{wt} \% \mathrm{GO}$ after reduction considerably increased to 34,22 , and $12.8 \mathrm{~S} / \mathrm{cm}$, respectively. This improvement in conductivity is attributed to the photoreduction of GO by CdS. It is discussed before that this resistance decrement is caused by elimination of hydroxyl groups in GO structure [47]. Furthermore, as it can be seen from Figure 10, by increasing the amount of GO up to $50 \%$ the conductivity of the films was decreased remarkably. This decrement is attributed to incomplete photo reduction process due to exorbitance increasing of GO sheets. The electrons, which are released from the surface of nanoparticles of CdS by irradiating visible light, are not enough to access all of the GO sheets and consequently reduce them. Therefore the CdS/GO ratio should be optimized in order to maximize the conductivity of prepared films. 


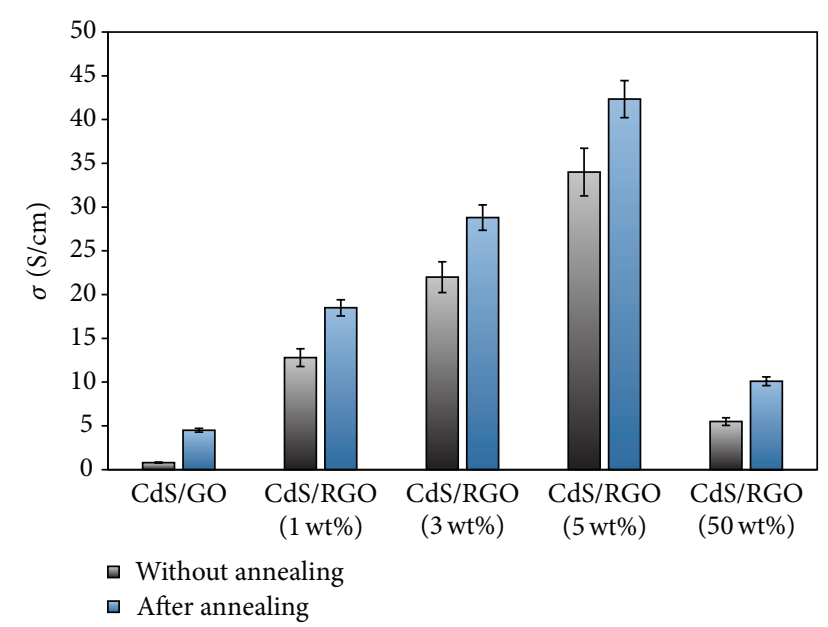

FIGURE 10: Effect of annealing and GO concentration on the conductivity of as-prepared films.

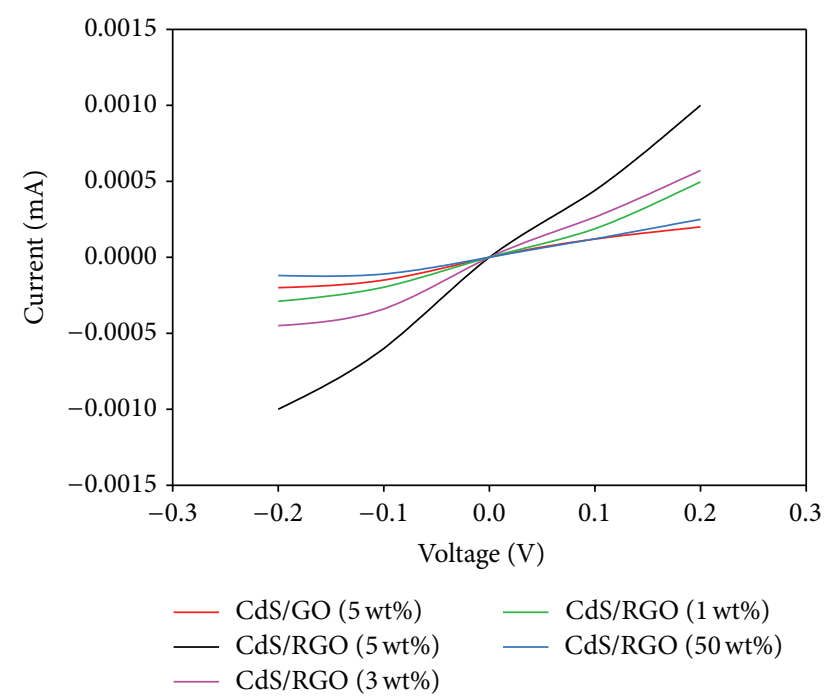

FIGURE 11: $I-V$ measurement of nanocomposites before (CdS/GO) and after (CdS/RGO) irradiation.

Annealing is generally known as a method to increase the conductivity of graphene films by further reduction of GO sheets [48]. The effect of the annealing is shown in Figure 10 (blue bar). As can be seen, annealing process leads to conductivity improvement of prepared films. The oxygencontaining functional groups were removed during annealing and caused decrease in the size of $\mathrm{sp}_{2}$ domains which can be further removed in this process [48].

Figure 11 shows the $I-V$ measurement of sensors with different ratio of graphene oxide in $\mathrm{CdS}$ solution, before $(\mathrm{CdS} / \mathrm{GO})$ and after (CdS/RGO) irradiation, is in good agreement with the 4-point probe observations. As it can be seen from voltage-current index, resistance of sensor with $\mathrm{CdS} / \mathrm{GO}$ composite (5 wt $\%$ ), before visible-light irradiation, is about $1000 \mathrm{~K} \Omega$ at a constant voltage $(0.2 \mathrm{~V})$ (Figure 11) which is much higher than the resistance of sensors after photoreduction. This decrement verifies photo reduction process. It is discussed before that this resistance decrement is caused by elimination of oxygene groups in GO structure. The $I-V$ characteristic of a sensor in which CdS/RGO (5 wt $\%)$, $\mathrm{CdS} / \mathrm{RGO}(3 \mathrm{wt} \%)$, and CdS/RGO (1 wt\%) are used as sensing materials after irradiation indicates that the resistance of sensors with this composite is about 210,324 , and $420 \mathrm{~K} \Omega$, respectively. But as it is shown in Figure 11, by increasing the amount of GO up to $50 \%$ the resistance of the sensor was increased remarkably (about $810 \mathrm{~K} \Omega$ ) which is attributed to incomplete photo reduction process due to exorbitance increasing GO sheets. The electrons which are released from the surface of nanoparticles of CdS by irradiating visible light are not enough to access all of the GO sheets and reduce them. Therefore the CdS/GO ratio should be optimized so that the maximum conductivity will be gained.

3.1. Effect of Annealing on Sensitivity of Gas Sensors. The sensitivity (normalized current) of the sensors before and after photoreduction and annealing plotted versus concentration of $\mathrm{CO}_{2}$ (ppm) is shown in Figure 12. As can be seen, by annealing the sensitivity of sensors is increased considerably which can contribute to the improvement in conductivity and the feasibility of electron mobility in surface area.

\subsection{Response of the Sensor with Optimized CdS/RGO} Nanocomposite. The $I-V$ changes of the sensors with optimized CdS/RGO thin film as sensing material were plotted versus concentration of $\mathrm{CO}_{2}(\mathrm{ppm})$ as shown in Figure 13. According to $I-V$ measurement, we find a response of $I_{\text {air }} / I_{\text {gas }} \sim 2.32$ at $1000 \mathrm{ppm} \mathrm{CO}_{2}$. Upon a $250 \mathrm{~s}$ exposure to $\mathrm{CO}_{2}$ gas, the sensors were found to exhibit a strong positive vapor coefficient behavior by giving a large change in current, followed by a recovery of over $250 \mathrm{~s}$ of purge with dry air. The response time of $\mathrm{CO}_{2}$ gas detection is about $10 \mathrm{~s}$. The response time is defined as the time taken for the relative conductance change to reach $90 \%$ of the steady-state value. The average recovery time is about $22 \mathrm{~s}$. The short recovery time can be attributed to the weak interaction between the $\mathrm{CO}_{2}$ gas molecules and graphene. The interaction of graphene with $\mathrm{CO}_{2}$ has many differences compared to the interaction of graphene with other gases (such as $\mathrm{NH}_{3}$ and $\mathrm{NO}_{2}$ ), and therefore high-temperature annealing in vacuum is not required to remove the adsorbed $\mathrm{CO}_{2}$ gas molecules $[5]$.

Furthermore, as can be seen from Figure 13, the sensors provide well sensitivity with high linearity and reversibility and results show that using solution of CdS/GO (5 wt\%) has more sensitivity due to its higher conductivity. Also the sensitivity of the sensors depends on the gas concentrations and improves by increasing the amount of gas concentrations from $200 \mathrm{ppm}$ to $1000 \mathrm{ppm}$. The $\mathrm{CO}_{2}$ gas molecule adsorption on a graphene sheet can induce an increase in graphene conductance. The $\mathrm{CO}_{2}$ gas molecule plays a donor/acceptor role on the graphene sheet. The graphene conductivity changes indicate a carrier transfer between $\mathrm{CO}_{2}$ gas and the graphene sheet. Therefore, the physical adsorption of $\mathrm{CO}_{2}$ gas on the graphene sheet plays an important role on the sensing mechanism. 


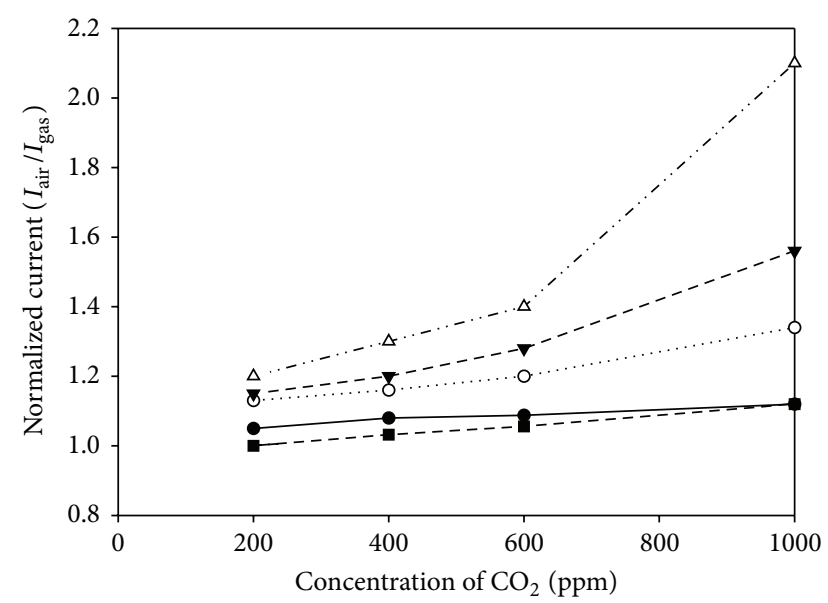

Before annealing

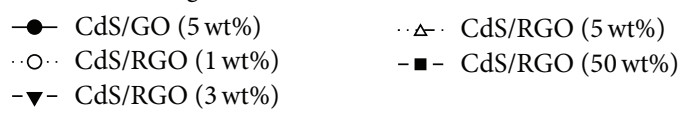

(a)

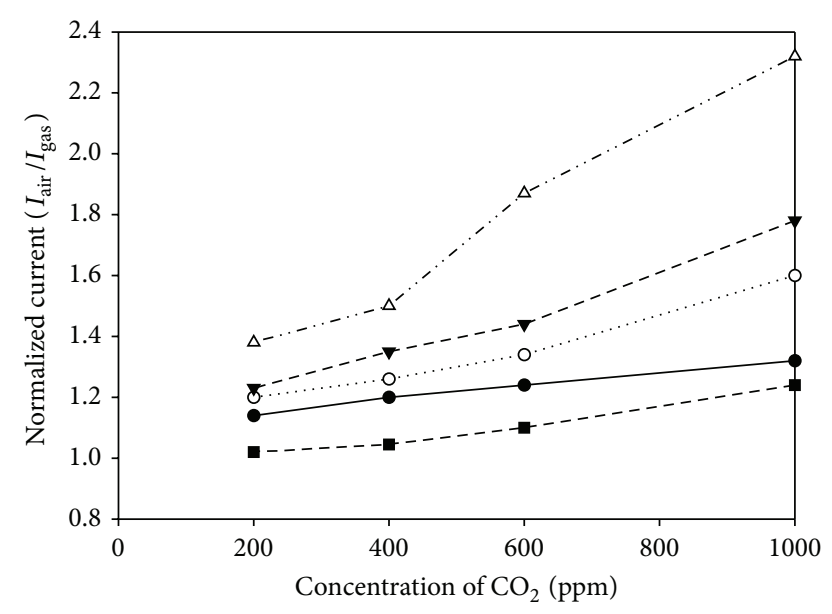

After annealing

$\rightarrow-\mathrm{CdS} / \mathrm{GO}(5 \mathrm{wt} \%)$
$-\mathrm{O}$ CdS/RGO (1wt\%)
- $-\mathrm{CdS} / \mathrm{RGO}(3 \mathrm{wt} \%)$

(b)

FIGURE 12: Sensitivity of gas sensors to various concentrations of $\mathrm{CO}_{2}$ and effect of annealing. (a) Before annealing and (b) after annealing.

\section{Conclusion}

In summary, CdS/RGO composites were successfully fabricated by a simple method. The electron transfer from excited $\mathrm{CdS}$ nanoparticles to GO is effective in carrying out reduction and decreasing the resistivity of chemically functionalized graphene films. The photocatalytic method offers a new and soft method of reduction as compared to the conventional approach of using elevated temperatures or strong reducing agents for reducing graphene oxide. In addition the binding of the oxide particles keeps the exfoliated graphene sheets from the collapse after the reduction step. Furthermore, our investigation confirms that annealing was not effective to

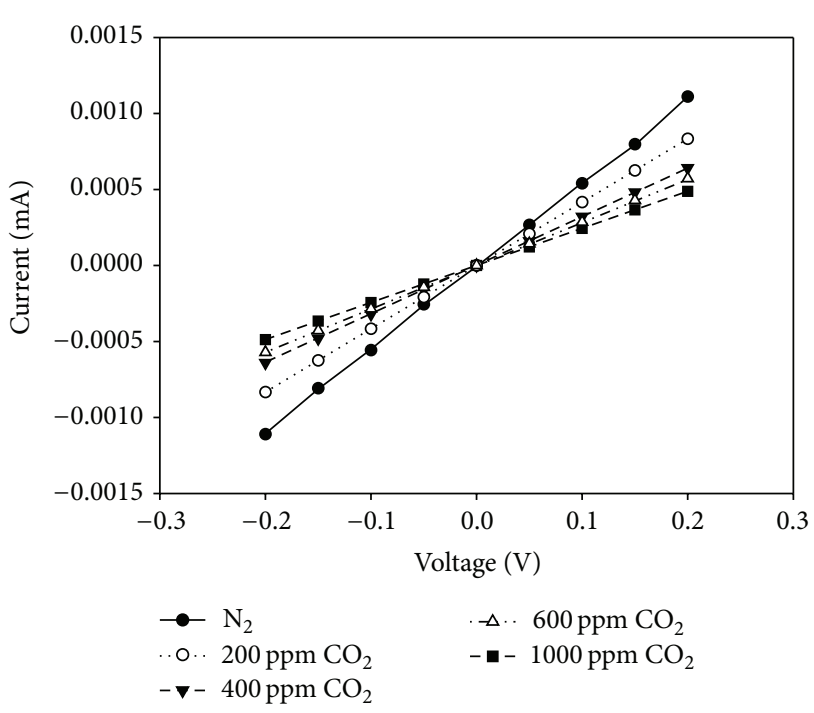

(a)

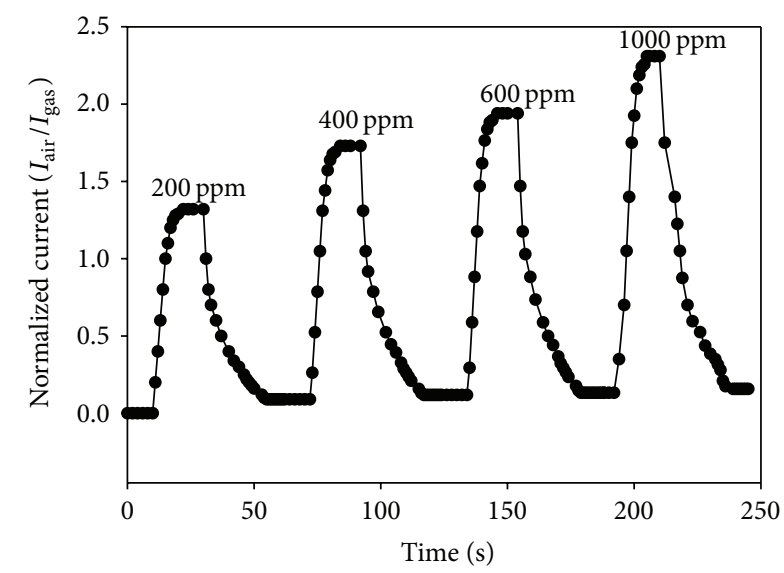

(b)

Figure 13: Sensitivity (a) and response (b) of gas sensors with optimized CdS/RGO nanocomposites to various concentrations of $\mathrm{CO}_{2}$.

remove oxygen groups which leads to conductivity improvement and also increase of the sensor performance. The promising application as a gas sensor for $\mathrm{CO}_{2}$ was also selected as a preliminary test of the electrocatalytic behavior of CdS nanoparticle/RGO composites. These sensors are low cost and show a rapid and highly sensitive response. In addition, in order to further study we examine the effect of $\mathrm{GO}$ concentration in reduction and device performance.

\section{Conflict of Interests}

The authors declare that there is no conflict of interests regarding the publication of this paper.

\section{Authors' Contribution}

Amirhossein Hasani and Hamed Sharifi Dehsari contributed equally to this work. 


\section{References}

[1] K. S. Novoselov, A. K. Geim, S. V. Morozov et al., "Electric field in atomically thin carbon films," Science, vol. 306, no. 5696, pp. 666-669, 2004.

[2] M. J. Allen, V. C. Tung, and R. B. Kaner, "Honeycomb carbon: a review of graphene," Chemical Reviews, vol. 110, no. 1, pp. 132$145,2010$.

[3] S. Park and R. S. Ruoff, "Chemical methods for the production of graphenes," Nature Nanotechnology, vol. 4, no. 4, pp. 217-224, 2009.

[4] A. K. Geim, “Graphene: status and prospects," Science, vol. 324, no. 5934, pp. 1530-1534, 2009.

[5] F. Schedin, A. K. Geim, S. V. Morozov et al., "Detection of individual gas molecules adsorbed on graphene," Nature Materials, vol. 6, no. 9, pp. 652-655, 2007.

[6] C. Berger, Z. Song, X. Li et al., "Electronic confinement and coherence in patterned epitaxial graphene," Science, vol. 312, no. 5777, pp. 1191-1196, 2006.

[7] P. W. Sutter, J.-I. Flege, and E. A. Sutter, "Epitaxial graphene on ruthenium," Nature Materials, vol. 7, no. 5, pp. 406-411, 2008.

[8] R. Ruoff, "Graphene: calling all chemists," Nature Nanotechnology, vol. 3, no. 1, pp. 10-11, 2008.

[9] C. K. Chua and M. Pumera, "Chemical reduction of graphene oxide: a synthetic chemistry viewpoint," Chemical Society Reviews, vol. 43, no. 1, pp. 291-312, 2014.

[10] X. Gao, J. Jang, and S. Nagase, "Hydrazine and thermal reduction of graphene oxide: reaction mechanisms, product structures, and reaction design," The Journal of Physical Chemistry C, vol. 114, no. 2, pp. 832-842, 2010.

[11] L. J. Cote, R. Cruz-Silva, and J. Huang, "Flash reduction and patterning of graphite oxide and its polymer composite," Journal of the American Chemical Society, vol. 131, no. 31, pp. 1102711032, 2009.

[12] W. Gao, N. Singh, L. Song et al., "Direct laser writing of microsupercapacitors on hydrated graphite oxide films," Nature Nanotechnology, vol. 6, no. 8, pp. 496-500, 2011.

[13] P. Zhou, H. Wei, Q. Sun et al., "The tunable electrical properties of graphene nano-bridges," Journal of Materials Chemistry C, vol. 1, no. 14, pp. 2548-2552, 2013.

[14] G. Williams, B. Seger, and P. V. Kamt, "TiO ${ }_{2}$-graphene nanocomposites. UV-assisted photocatalytic reduction of graphene oxide," ACS Nano, vol. 2, no. 7, pp. 1487-1491, 2008.

[15] T. Wu, S. Liu, Y. Luo, W. Lu, L. Wang, and X. Sun, "Surface plasmon resonance-induced visible light photocatalytic reduction of graphene oxide: using Ag nanoparticles as a plasmonic photocatalyst," Nanoscale, vol. 3, no. 5, pp. 2142-2144, 2011.

[16] L. Brus, "Electronic wave functions in semiconductor clusters: experiment and theory," Journal of Physical Chemistry, vol. 90, no. 12, pp. 2555-2560, 1986.

[17] A. P. Alivisatos, "Perspectives on the physical chemistry of semiconductor nanocrystals," The Journal of Physical Chemistry, vol. 100, no. 31, pp. 13226-13239, 1996.

[18] R. Mahtab, J. P. Rogers, C. P. Singleton, and C. J. Murphy, "Preferential adsorption of a "kinked" DNA to a neutral curved surface: comparisons to and implications for nonspecific DNAprotein interactions," Journal of the American Chemical Society, vol. 118, no. 30, pp. 7028-7032, 1996.

[19] M. Bruchez Jr., M. Moronne, P. Gin, S. Weiss, and A. P. Alivisatos, "Semiconductor nanocrystals as fluorescent biological labels," Science, vol. 281, no. 5385, pp. 2013-2016, 1998.
[20] Y. Ye, L. Gan, L. Dai et al., "Multicolor graphene nanoribbon/semiconductor nanowire heterojunction light-emitting diodes," Journal of Materials Chemistry, vol. 21, no. 32, pp. 11760$11763,2011$.

[21] T. Dufaux, J. Boettcher, M. Burghard, and K. Kern, "Photocurrent distribution in graphene-CdS nanowire devices," Small, vol. 6, no. 17, pp. 1868-1872, 2010.

[22] L.-L. Li, K.-P. Liu, G.-H. Yang, C.-M. Wang, J.-R. Zhang, and J.J. Zhu, "Fabrication of graphene-quantum dots composites for sensitive electrogenerated chemiluminescence immunosensing," Advanced Functional Materials, vol. 21, no. 5, pp. 869-878, 2011.

[23] P. Wang, T. Jiang, C. Zhu, Y. Zhai, D. Wang, and S. Dong, "Onestep, solvothermal synthesis of graphene-CdS and graphene$\mathrm{ZnS}$ quantum dot nanocomposites and their interesting photovoltaic properties," Nano Research, vol. 3, no. 11, pp. 794-799, 2010.

[24] H. Chang, X. Lv, H. Zhang, and J. Li, "Quantum dots sensitized graphene: in situ growth and application in photoelectrochemical cells," Electrochemistry Communications, vol. 12, no. 3, pp. 483-487, 2010.

[25] L. Jia, D. H. Wang, Y. X. Huang, A. W. Xu, and H. Q. Yu, "Highly durable N-doped graphene/CdS nanocomposites with enhanced photocatalytic hydrogen evolution from water under visible light irradiation," The Journal of Physical Chemistry C, vol. 115, no. 23, pp. 11466-11473, 2011.

[26] Q. Li, B. Guo, J. Yu et al., "Highly efficient visible-light-driven photocatalytic hydrogen production of CdS-cluster-decorated graphene nanosheets," Journal of the American Chemical Society, vol. 133, no. 28, pp. 10878-10884, 2011.

[27] Y. Liu, X. Dong, and P. Chen, "Biological and chemical sensors based on graphene materials," Chemical Society Reviews, vol. 41, no. 6, pp. 2283-2307, 2012.

[28] C.-L. Sun, C.-T. Chang, H.-H. Lee et al., "Microwave-assisted synthesis of a core-shell MWCNT/GONR heterostructure for the electrochemical detection of ascorbic acid, dopamine, and uric acid," ACS Nano, vol. 5, no. 10, pp. 7788-7795, 2011.

[29] F. Xiao, J. Song, H. Gao, X. Zan, R. Xu, and H. Duan, "Coating graphene paper with 2D-assembly of electrocatalytic nanoparticles: a modular approach toward high-performance flexible electrodes," ACS Nano, vol. 6, no. 1, pp. 100-110, 2012.

[30] X. An, X. Yu, J. C. Yu, and G. Zhang, “CdS nanorods/reduced graphene oxide nanocomposites for photocatalysis and electrochemical sensing," Journal of Materials Chemistry A, vol. 1, no. 16, pp. 5158-5164, 2013.

[31] H. J. Yoon, D. H. Jun, J. H. Yang, Z. Zhou, S. S. Yang, and M. M.C. Cheng, "Carbon dioxide gas sensor using a graphene sheet," Sensors and Actuators B, vol. 157, pp. 310-313, 2011.

[32] P. G. Collins, K. Bradley, M. Ishigami, and A. Zettl, "Extreme oxygen sensitivity of electronic properties of carbon nanotubes," Science, vol. 287, no. 5459, pp. 1801-1804, 2000.

[33] J. Kong, M. G. Chapline, and H. Dai, "Functionalized carbon nanotubes for molecular hydrogen sensors," Advanced Materials, vol. 13, no. 18, pp. 1384-1386, 2001.

[34] K. G. Ong, K. Zeng, and C. A. Grimes, "A wireless, passive carbon nanotube-based gas sensor," IEEE Sensors Journal, vol. 2, no. 2, pp. 82-88, 2002.

[35] C. Cantalini, L. Valentini, L. Lozzi, I. Armentano, J. M. Kenny, and S. Santucci, " $\mathrm{NO}_{2}$ gas sensitivity of carbon nanotubes obtained by plasma enhanced chemical vapor deposition," Sensors and Actuators, B: Chemical, vol. 93, no. 1-3, pp. 333-337, 2003. 
[36] S. Chopra, K. McGuire, N. Gothard, A. M. Rao, and A. Pham, "Selective gas detection using a carbon nanotube sensor," Applied Physics Letters, vol. 83, no. 11, pp. 2280-2282, 2003.

[37] S. G. Wang, Q. Zhang, D. J. Yang, P. J. Sellin, and G. F. Zhong, "Multi-walled carbon nanotube-based gas sensors for $\mathrm{NH}_{3}$ detection," Diamond and Related Materials, vol. 13, no. 4-8, pp. 1327-1332, 2004.

[38] J. Suehiro, G. Zhou, H. Imakiire, W. Ding, and M. Hara, "Controlled fabrication of carbon nanotube $\mathrm{NO}_{2}$ gas sensor using dielectrophoretic impedance measurement," Sensors and Actuators, B: Chemical, vol. 108, no. 1-2, pp. 398-403, 2005.

[39] H. S. Dehsari, E. K. Shalamzari, J. N. Gavgani, F. A. Taromi, and S. Ghanbary, "Efficient preparation of ultralarge graphene oxide using a PEDOT:PSS/GO composite layer as hole transport layer in polymer-based optoelectronic devices," RSC Advances, vol. 4, no. 98, pp. 55067-55076, 2014.

[40] N. R. Wilson, P. A. Pandey, R. Beanland et al., "Graphene oxide: structural analysis and application as a highly transparent support for electron microscopy," ACS Nano, vol. 3, no. 9, pp. 2547-2556, 2009.

[41] A. C. Ferrari, J. C. Meyer, V. Scardaci et al., "Raman spectrum of graphene and graphene layers," Physical Review Letters, vol. 97, no. 18, Article ID 187401, 2006.

[42] X. C. Dong, Y. M. Shi, Y. Zhao et al., "Symmetry breaking of graphene monolayers by molecular decoration," Physical Review Letters, vol. 102, no. 13, Article ID 135501, 2009.

[43] G. Williams and P. V. Kamat, "Graphene-semiconductor nanocomposites: excited-state interactions between $\mathrm{ZnO}$ nanoparticles and graphene oxide," Langmuir, vol. 25, no. 24, pp. 13869-13873, 2009.

[44] O. Akhavan, "Photocatalytic reduction of graphene oxides hybridized by $\mathrm{ZnO}$ nanoparticles in ethanol," Carbon, vol. 49, no. 1, pp. 11-18, 2011.

[45] P. V. Kamat, I. Bedja, and S. Hotchandani, "Photoinduced charge transfer between carbon and semiconductor clusters. One-electron reduction of $\mathrm{C} 60$ in colloidal $\mathrm{TiO}_{2}$ semiconductor suspensions," Journal of Physical Chemistry, vol. 98, no. 37, pp. 9137-9142, 1994.

[46] A. Kongkanand and P. V. Kamat, "Electron storage in single wall carbon nanotubes. Fermi level equilibration in semiconductorSWCNT suspensions," ACS Nano, vol. 1, no. 1, pp. 13-21, 2007.

[47] H. Li and C. Bubeck, "Photoreduction processes of graphene oxide and related applications," Macromolecular Research, vol. 21, no. 3, pp. 290-297, 2013.

[48] C.-M. Chena, J.-Q. Huangb, Q. Zhang et al., "Annealing a graphene oxide film to produce a free standing high conductive graphene film," Carbon, vol. 50, no. 2, pp. 659-667, 2012. 

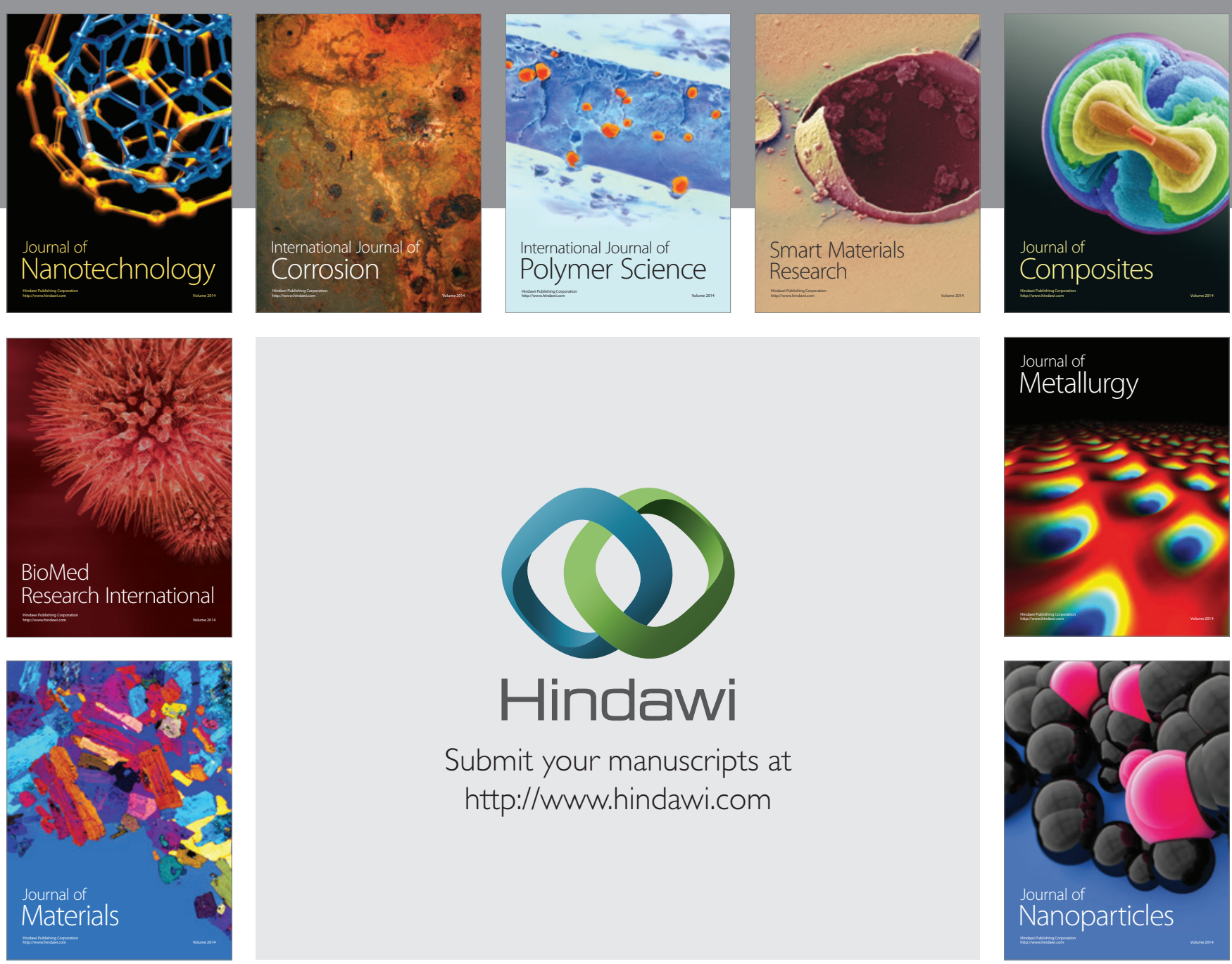

Submit your manuscripts at http://www.hindawi.com
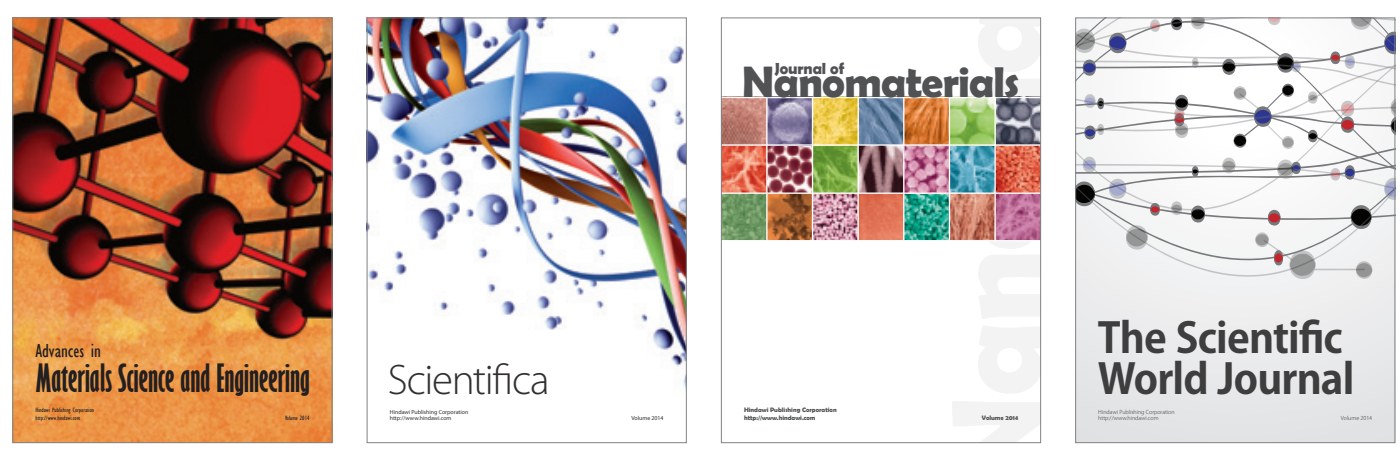

\section{The Scientific World Journal}
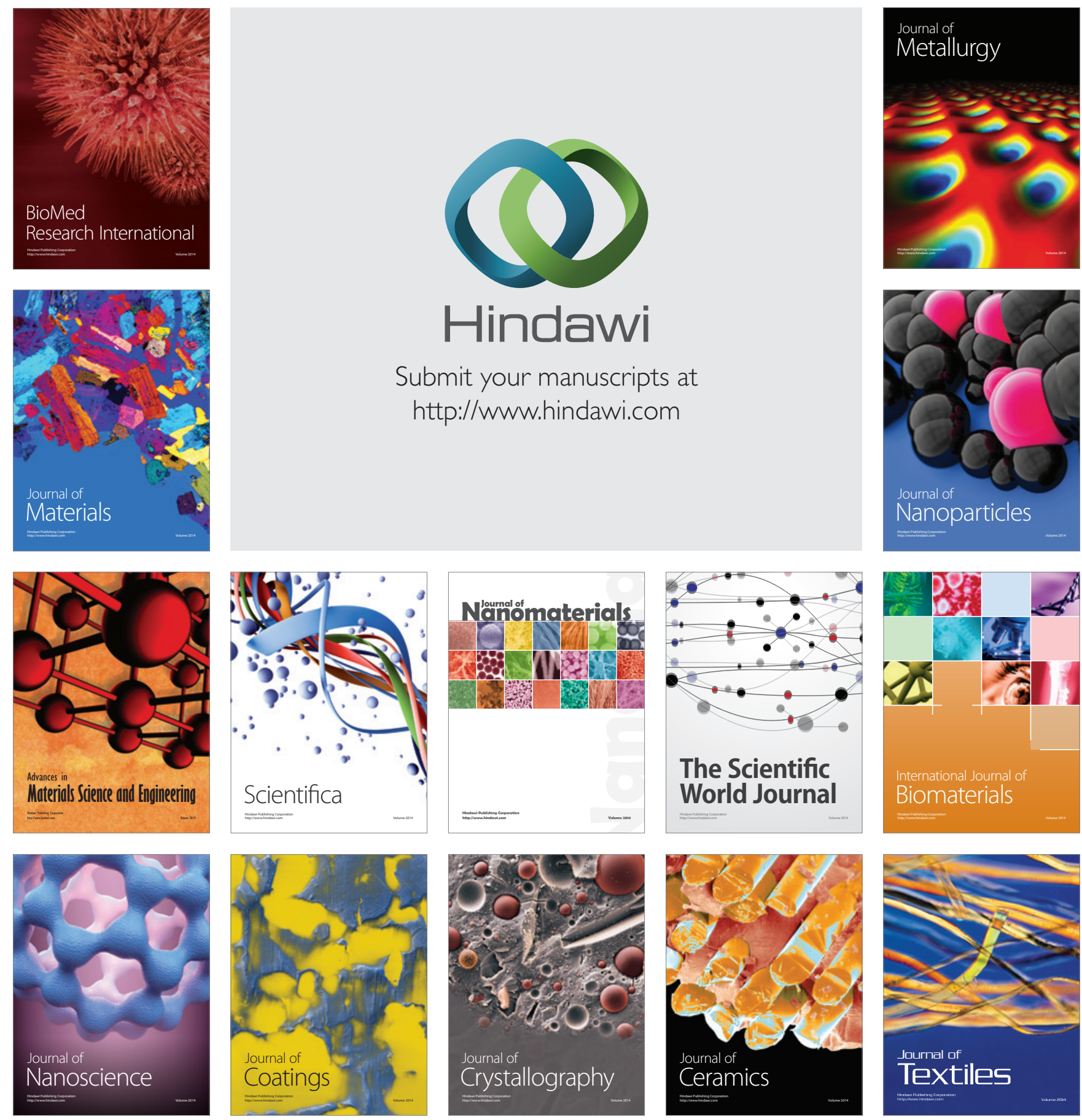